TRANSACTIONS OF THE

AMERICAN MATHEMATICAL SOCIETY

Volume 358, Number 4, Pages 1705-1714

S 0002-9947(05)03789-X

Article electronically published on September 22, 2005

\title{
POISSON STRUCTURES ON COMPLEX FLAG MANIFOLDS ASSOCIATED WITH REAL FORMS
}

\author{
PHILIP FOTH AND JIANG-HUA LU \\ Dedicated to Alan Weinstein on the occasion of his 60th birthday
}

\begin{abstract}
For a complex semisimple Lie group $G$ and a real form $G_{0}$ we define a Poisson structure on the variety of Borel subgroups of $G$ with the property that all $G_{0}$-orbits in $X$ as well as all Bruhat cells (for a suitable choice of a Borel subgroup of $G$ ) are Poisson submanifolds. In particular, we show that every non-empty intersection of a $G_{0}$-orbit and a Bruhat cell is a regular Poisson manifold, and we compute the dimension of its symplectic leaves.
\end{abstract}

\section{INTRODUCTION}

Let $G$ be a connected and simply-connected complex semisimple Lie group with Lie algebra $\mathfrak{g}$, and let $X$ be the variety of Borel subalgebras of $\mathfrak{g}$. In this paper we use a real form $\mathfrak{g}_{0}$ of $\mathfrak{g}$ to define a Poisson structure on $X$. This Poisson structure depends on a choice of a Borel subalgebra $\mathfrak{b}$ of $\mathfrak{g}$ such that $\mathfrak{g}_{0} \cap \mathfrak{b}$ is a maximally compact Cartan subalgebra of $\mathfrak{g}_{0}$. Instead of dealing with each real form individually, we fix a Borel subalgebra $\mathfrak{b}$ of $\mathfrak{g}$ and a Cartan subalgebra $\mathfrak{h} \subset \mathfrak{b}$. Then, as is shown in [6, a real form $\mathfrak{g}_{v}$ of $\mathfrak{g}$ can be constructed from each Vogan diagram $v$ for $\mathfrak{g}$ such that $\mathfrak{g}_{v} \cap \mathfrak{b}$ is a maximally compact Cartan subalgebra of $\mathfrak{g}_{v}$. The corresponding Poisson structure on $X$ is denoted by $\Pi_{v}$.

Let $G_{v}$ be the real form of $G$ corresponding to $\mathfrak{g}_{v}$, and let $B$ be the Borel subgroup of $G$ with Lie algebra $\mathfrak{b}$. The Poisson structure $\Pi_{v}$ has the property that each $G_{v}$-orbit as well as each $B$-orbit in $X$ is a Poisson submanifold. The $B$-orbits in $X$ will be referred to as the Bruhat cells. We compute the rank of $\Pi_{v}$. In particular, if a $G_{v}$-orbit $\mathcal{O}$ meets a Bruhat cell $\mathcal{C}$, they intersect transversally, and we find that all the symplectic leaves in $\mathcal{O} \cap \mathcal{C}$ have the same dimension, so $\mathcal{O} \cap \mathcal{C}$ is a regular Poisson manifold. Moreover, we show that all symplectic leaves in each connected component of $\mathcal{O} \cap \mathcal{C}$ are translates of each other by elements of a Cartan subgroup of $G_{v}$. We also show that the $G_{v}$-invariant Poisson cohomology for each open $G_{v}$-orbit in $X$ is isomorphic to the de Rham cohomology of $X$.

Results similar to those presented here for the full flag manifold $X=G / B$ are also valid for a partial flag manifold $G / P$, where $P$ is a parabolic subgroup of

Received by the editors September 30, 2003 and, in revised form, June 16, 2004.

2000 Mathematics Subject Classification. Primary 53D17; Secondary 14M15, 22 E15.

Key words and phrases. Lie groups, real forms, flag varieties, Poisson structures, symplectic leaves.

(C)2005 American Mathematical Society

Reverts to public domain 28 years from publication 1705 
$G$ containing $B$. We will treat these more general cases as well as some further properties of $\Pi_{v}$ in a future paper.

Throughout this paper, if $V$ is a set and $\sigma$ is an involution on $V$, we will use $V^{\sigma}$ to denote the fixed point set of $\sigma$ in $V$.

\section{REAL FORMS OF $\mathfrak{g}$ AND VOGAN DiAgRAMS}

Let $\mathfrak{g}$ be a complex simple Lie algebra. In this section we recall the classification of real forms of $\mathfrak{g}$ by Vogan diagrams. Details can be found in [6, Chapter 6].

Suppose that $\mathfrak{g}_{0}$ is a real form of $\mathfrak{g}$ and that $\tau_{0}$ is the corresponding complexconjugate linear involution on $\mathfrak{g}$. Let $\theta_{0}$ be a Cartan involution of $\mathfrak{g}_{0}$, and let $\mathfrak{h}_{0}$ be a $\theta_{0}$-stable maximally compact Cartan subalgebra of $\mathfrak{g}_{0}$. Set $\mathfrak{t}_{0}=\mathfrak{h}_{0}^{\theta_{0}}$ and $\mathfrak{a}_{0}=\mathfrak{h}_{0}^{-\theta_{0}}$ so that $\mathfrak{h}_{0}=\mathfrak{t}_{0}+\mathfrak{a}_{0}$. Let $\gamma_{0}$ be the complexification of $\theta_{0}$. Then the Cartan subalgebra $\mathfrak{h}=\mathfrak{h}_{0}+i \mathfrak{h}_{0}$ of $\mathfrak{g}$ is $\gamma_{0}$-stable. Let $\Delta$ be the root system for $(\mathfrak{g}, \mathfrak{h})$. Since $\mathfrak{h}_{0}$ is a maximally compact Cartan subalgebra of $\mathfrak{g}_{0}$, there exists $x_{0} \in i \mathfrak{t}_{0}$ that is regular for $\Delta$. Define the subset $\Delta^{+}$of positive roots in $\Delta$ by $\alpha \in \Delta^{+}$if and only if $\alpha\left(x_{0}\right)>0$. Then $\gamma_{0}\left(\Delta^{+}\right)=\Delta^{+}$. Let $\Sigma \subset \Delta^{+}$be the set of simple roots in $\Delta^{+}$. Then $\gamma_{0}(\Sigma)=\Sigma$, so $\gamma_{0}$ gives rise to an involutive automorphism of the Dynkin diagram of $\mathfrak{g}$. Let $\mathcal{I}$ be the set of non-compact imaginary simple roots. The Vogan diagram of $\mathfrak{g}_{0}$ associated to the triple $\left(\theta_{0}, \mathfrak{h}_{0}, \Delta^{+}\right)$is the Dynkin diagram $D(\mathfrak{g})$ of $\mathfrak{g}$, together with an involutive automorphism $\gamma_{0}$ on $D(\mathfrak{g})$ and the vertices corresponding to the simple roots in $\mathcal{I}$ painted black.

In general, a Vogan diagram for $\mathfrak{g}$ is defined to be a triple $(D(\mathfrak{g}), d, \mathcal{I})$, where $D(\mathfrak{g})$ is the Dynkin diagram of $\mathfrak{g}, d$ is an involutive automorphism of $D(\mathfrak{g})$, and $\mathcal{I}$ is a subset of vertices of $D(\mathfrak{g})$ such that $d(\alpha)=\alpha$ for each $\alpha \in \mathcal{I}$. Every Vogan diagram for $\mathfrak{g}$ comes from a real form of $\mathfrak{g}$ (see below), although two different Vogan diagrams can come from isomorphic real forms. A non-redundant list of Vogan diagrams with the corresponding isomorphism class of real forms for all simple Lie algebras is given in [6]. Every Vogan diagram in the list in [6] is normalized in the sense that at most one vertex is painted black.

For the purpose of defining Poisson structures on the variety of Borel subalgebras of $\mathfrak{g}$, we now recall the explicit construction of a real form of $\mathfrak{g}$ from a Vogan diagram [6. Theorem 6.88]. We need to fix the following data for $\mathfrak{g}$.

Choose a Cartan subalgebra $\mathfrak{h}$ of $\mathfrak{g}$ and let $\Delta$ be the root system for $(\mathfrak{g}, \mathfrak{h})$. Fix a choice of positive roots $\Delta^{+}$and let $\Sigma$ be the basis of simple roots. Let $\langle\langle\rangle$, be the Killing form of $\mathfrak{g}$ and let root vectors $\left\{E_{\alpha}: \alpha \in \Delta\right\}$ be chosen such that $\left[E_{\alpha}, E_{-\alpha}\right]=H_{\alpha}$ for each $\alpha \in \Delta^{+}$, where $H_{\alpha}$ is the unique element of $\mathfrak{h}$ defined by $\left\langle\left\langle H, H_{\alpha}\right\rangle\right\rangle=\alpha(H)$ for all $H \in \mathfrak{h}$, and such that the numbers $m_{\alpha, \beta}$ given by $\left[E_{\alpha}, E_{\beta}\right]=m_{\alpha, \beta} E_{\alpha+\beta}$ when $\alpha+\beta \in \Delta$ are real. Define a compact real form $\mathfrak{k}$ of $\mathfrak{g}$ as

$$
\mathfrak{k}=\operatorname{span}_{\mathbb{R}}\left\{i H_{\alpha}, X_{\alpha}:=E_{\alpha}-E_{-\alpha}, Y_{\alpha}:=i\left(E_{\alpha}+E_{-\alpha}\right)\right\},
$$

and let $\theta$ be the complex conjugation of $\mathfrak{g}$ defining $\mathfrak{k}$. If $d$ is an involutive automorphism of the Dynkin diagram of $\mathfrak{g}$, define $\gamma_{d}$ to be the unique automorphism of $\mathfrak{g}$ satisfying $\gamma_{d}\left(H_{\alpha}\right)=H_{d(\alpha)}$ and $\gamma_{d}\left(E_{\alpha}\right)=E_{d(\alpha)}$ for each simple root $\alpha$.

Given a Vogan diagram $v$ for $\mathfrak{g}$, not necessarily normalized, with the involutive diagram automorphism $d$, let $t_{v}$ be the unique element in the adjoint group of $\mathfrak{g}$ such that

$$
\operatorname{Ad}_{t_{v}}\left(E_{\alpha}\right)=\left\{\begin{array}{l}
E_{\alpha} \text { if } \alpha \text { is a blank vertex in } v, \\
-E_{\alpha} \text { if } \alpha \text { is a painted vertex in } v .
\end{array}\right.
$$


Define a complex conjugate linear involution

$$
\tau_{v}:=\operatorname{Ad}_{t_{v}} \circ \gamma_{d} \circ \theta
$$

Notation 2.1. We use $\mathfrak{g}_{v}=\mathfrak{g}^{\tau_{v}}$ to denote the real form of $\mathfrak{g}$ defined by $\tau_{v}$. Set $\theta_{v}=\left.\theta\right|_{\mathfrak{g}_{v}}$. Then $\theta_{v}$ is a Cartan involution of $\mathfrak{g}_{v}$, and $\mathfrak{h}^{\tau_{v}}$ is a $\theta_{v}$-stable maximally compact Cartan subalgebra of $\mathfrak{g}_{v}$, with $\mathfrak{h}=\mathfrak{h}^{\tau_{v}}+i \mathfrak{h}^{\tau_{v}}$. The complexification of $\tau_{v}$ is

$$
\gamma_{v}:=\tau_{v} \theta=\theta \tau_{v}=\operatorname{Ad}_{t_{v}} \gamma_{d}
$$

Since $\gamma_{v}\left(\Delta^{+}\right)=\Delta^{+}$, the Vogan diagram of $\mathfrak{g}_{v}$ associated to the triple $\left(\theta_{v}, \mathfrak{h}^{\tau_{v}}, \Delta^{+}\right)$ is $v$.

One of the advantages of introducing the real form $\mathfrak{g}_{v}$ is as follows. We say that a real subalgebra $\mathfrak{l}$ of $\mathfrak{g}$ is Lagrangian if its real dimension is equal to the complex dimension of $\mathfrak{g}$ and if $\operatorname{Im}\left\langle\left\langle x_{1}, x_{2}\right\rangle\right\rangle=0$ for all $x_{1}, x_{2} \in \mathfrak{l}$. A decomposition $\mathfrak{g}=\mathfrak{l}_{1}+\mathfrak{l}_{2}$ is called a Lagrangian splitting if both $\mathfrak{l}_{1}$ and $\mathfrak{l}_{2}$ are Lagrangian. Let $\mathfrak{n}$ be the subalgebra of $\mathfrak{g}$ spanned by the set of all positive root vectors for $\Delta^{+}$. The following fact is easy to prove.

Lemma 2.2. Let $\mathfrak{l}_{d}:=\mathfrak{h}^{-\tau_{v}}+\mathfrak{n}$. Then $\mathfrak{g}=\mathfrak{g}_{v}+\mathfrak{l}_{d}$ is a Lagrangian splitting of $\mathfrak{g}$.

Let $\mathfrak{a}=\operatorname{span}_{\mathbb{R}}\left\{i H_{\alpha}: \alpha \in \Sigma\right\}$, and let $\mathfrak{t}=i \mathfrak{a}$. We note that since

$$
\mathfrak{h}^{-\tau_{v}}=\mathfrak{h}^{-\gamma_{d} \circ \theta}=\mathfrak{t}^{-\gamma_{d}}+\mathfrak{a}^{\gamma_{d}},
$$

the Lagrangian complement $\mathfrak{l}_{d}$ of $\mathfrak{g}_{v}$ depends only on $d$, and in the case when $d=1$, we have $\mathfrak{l}_{d}=\mathfrak{a}+\mathfrak{n}$. Note that $\mathfrak{h}^{\tau_{v}}=\mathfrak{h}^{\gamma_{d} \circ \theta}=\mathfrak{t}^{\gamma_{d}}+\mathfrak{a}^{-\gamma_{d}}$ also depends only on $d$.

Remark 2.3. Recall [2, Definition 6.10] that two real forms $\tau_{1}$ and $\tau_{2}$ are said to be in the same inner class if there exists $g \in \operatorname{Int}(\mathfrak{g})$, the adjoint group of $\mathfrak{g}$, such that $\tau_{1}=\operatorname{Ad}_{g} \tau_{2}$. Inner classes of real forms are in one-to-one correspondence with involutive automorphisms of the Dynkin diagram of $\mathfrak{g}$ [2, Proposition 6.12]. Let $d$ be an involutive automorphism of $D(\mathfrak{g})$. Then as $v$ runs over the collection of all Vogan diagrams with $d$ as the diagram automorphism, the real form $\mathfrak{g}_{v}$ runs over all $\operatorname{Int}(\mathfrak{g})$-conjugacy classes of real forms of $\mathfrak{g}$ in the inner class corresponding to $d$.

\section{The Poisson structure $\Pi_{v}$ on $X$}

Let $\mathfrak{g}$ be a complex semi-simple Lie algebra, and let $X$ be the variety of all Borel subalgebras of $\mathfrak{g}$. We keep the notation from Section 2, Let $v$ be a Vogan diagram for $\mathfrak{g}$ and let $\mathfrak{g}_{v}=\mathfrak{g}^{\tau_{v}}$ be the real form of $\mathfrak{g}$ constructed in Section 2, Let $G$ be the connected and simply-connected Lie group with Lie algebra $\mathfrak{g}$. Without any risk of confusion, we shall also denote by $\tau_{v}$ the lift of $\tau_{v}$ from $\mathfrak{g}$ to $G$, and we set $G_{v}=G^{\tau_{v}}$. It follows from [5, Theorem 8.2, p. 320] that the group $G_{v}$ is connected.

In this section, we will start with a Vogan diagram $v$ for $\mathfrak{g}$ and define a Poisson structure $\Pi_{v}$ on $X$ such that every $G_{v}$-orbit in $X$ is a Poisson submanifold. This Poisson structure comes from an identification of $X$ with the $G$-orbit through $\mathfrak{t}+\mathfrak{n}$ inside the variety $\mathcal{L}$ of Lagrangian subalgebras of $\mathfrak{g}$, which was studied in [3]. We now recall the relevant details.

Set $n=\operatorname{dim}_{\mathbb{C}} \mathfrak{g}$ and let $\operatorname{Gr}_{\mathbb{R}}(n, \mathfrak{g})$ be the Grassmannian of real $n$-dimensional subspaces of $\mathfrak{g}$. The set $\mathcal{L}$ of all Lagrangian subalgebras of $\mathfrak{g}$ is naturally a real subvariety of $\operatorname{Gr}_{\mathbb{R}}(n, \mathfrak{g})$. The natural action of $G$ on $\operatorname{Gr}_{\mathbb{R}}(n, \mathfrak{g})$ gives rise to a Lie algebra anti-homomorphism $\kappa$ from $\mathfrak{g}$ to the Lie algebra of vector fields on $\operatorname{Gr}_{\mathbb{R}}(n, \mathfrak{g})$, 
whose extension from $\wedge^{2} \mathfrak{g}$ to the space of bi-vector fields on $\operatorname{Gr}_{\mathbb{R}}(n, \mathfrak{g})$ will also be denoted by $\kappa$. Given a Lagrangian splitting $\mathfrak{g}=\mathfrak{l}_{1}+\mathfrak{l}_{2}$, we define the element $R_{\mathfrak{l}_{1}, \mathfrak{l}_{2}} \in \bigwedge^{2} \mathfrak{g}$ by

$$
\left\langle R_{\mathfrak{l}_{1}, \mathfrak{l}_{2}},\left(x_{1}+\xi_{1}\right) \wedge\left(x_{2}+\xi_{2}\right)\right\rangle=\left\langle\xi_{2}, x_{1}\right\rangle-\left\langle\xi_{1}, x_{2}\right\rangle, \quad x_{1}, x_{2} \in \mathfrak{l}_{1}, \xi_{1}, \xi_{2} \in \mathfrak{l}_{2},
$$

where $\langle\rangle=,\operatorname{Im}\langle\langle\rangle$,$\rangle . Set \Pi_{\mathfrak{l}_{1}, \mathfrak{l}_{2}}=\frac{1}{2} \kappa\left(R_{\mathfrak{l}_{1}, \mathfrak{l}_{2}}\right)$. Clearly, $\Pi_{\mathfrak{l}_{1}, \mathfrak{l}_{2}}$ is tangent to every $G$-orbit in $\operatorname{Gr}_{\mathbb{R}}(n, \mathfrak{g})$, so it is tangent to $\mathcal{L}$.

Theorem 3.1 ([3, Theorems 2.14 and 2.18]). The bi-vector field $\Pi_{\mathfrak{l}_{1}, \mathfrak{l}_{2}}$ restricts to a Poisson structure on $\mathcal{L}$. If $L_{1}$ and $L_{2}$ are the connected subgroups of $G$ with Lie algebras $\mathfrak{l}_{1}$ and $\mathfrak{l}_{2}$ respectively, then all the $L_{1}$ - as well as $L_{2}$-orbits in $\mathcal{L}$ are Poisson submanifolds with respect to $\Pi_{\mathfrak{l}_{1}, \mathfrak{l}_{2}}$.

For $\mathfrak{l} \in \mathcal{L}$, let $\mathfrak{n}(\mathfrak{l})$ be the normalizer subalgebra of $\mathfrak{l}$ in $\mathfrak{l}_{1}$. Let $\mathfrak{m}(\mathfrak{l})$ be the annihilator of $\mathfrak{n}(\mathfrak{l})$ in ll, i.e. $\mathfrak{m}(\mathfrak{l})=\{x \in \mathfrak{l}:\langle x, y\rangle=0 \forall y \in \mathfrak{n}(\mathfrak{l})\} \subset \mathfrak{l}$, and let $\mathcal{V}(\mathfrak{l})=\mathfrak{n}(\mathfrak{l})+\mathfrak{m}(\mathfrak{l})$.

Proposition 3.2 ([3, Theorem 2.21], [9, Corollary 7.3]). For each $\mathfrak{l} \in \mathcal{L}$, the space $\mathcal{V}(\mathfrak{l})$ is a Lagrangian subalgebra of $\mathfrak{g}$. The co-dimension of the symplectic leaf of $\Pi_{\mathfrak{l}_{1}, \mathfrak{l}_{2}}$ through $\mathfrak{l}$ in the orbit $L_{1} \cdot \mathfrak{l}$ is equal to $\operatorname{dim}\left(\mathcal{V}(\mathfrak{l}) \cap \mathfrak{l}_{2}\right)$.

Notation 3.3. Let $v$ be a Vogan diagram for $\mathfrak{g}$. We denote by $\Pi_{v}$, the Poisson structure on $\mathcal{L}$ defined by the Lagrangian splitting $\mathfrak{g}=\mathfrak{g}_{v}+\mathfrak{l}_{d}$ in Lemma 2.2. Let $H, N$, and $B$ be respectively the connected subgroups of $G$ with Lie algebras $\mathfrak{h}$, $\mathfrak{n}$, and $\mathfrak{b}=\mathfrak{h}+\mathfrak{n}$, so $B=H N$. Identify the $G$-orbit through $\mathfrak{t}+\mathfrak{n} \in \mathcal{L}$ with $G / B \cong X$. The induced Poisson structure on $X$ will also be denoted by $\Pi_{v}$. Let $H^{-\gamma_{d} \circ \theta}=\left\{h \in H: \gamma_{d} \circ \theta(h)=h^{-1}\right\}$ and let $L_{d}=H^{-\gamma_{d} \circ \theta} N$. By the Bruhat lemma, orbits of $L_{d}$ in $X \cong G / B$, which are the same as the $N$-orbits in $X$, are labeled by the elements in the Weyl group $W$ of $\Delta$. We refer to these $N$-orbits as the Bruhat cells in $X$.

By [3, Theorem 2.18], we have

Proposition 3.4. Each $G_{v}$-orbit in $X$ as well as each Bruhat cell in $X$ is a Poisson submanifold with respect to $\Pi_{v}$.

When $v$ is the Vogan diagram with $d=1$ and no vertex painted, we have $\tau_{v}=\theta$, so $\mathfrak{g}_{v}=\mathfrak{k}$. The Poisson structure $\Pi_{v}$ in this case was first introduced in [11] and 13, and it has the property that its symplectic leaves are precisely the Bruhat cells (hence the name "Bruhat Poisson structure" in [11]). In [3] and [10] this Poisson structure was related to some earlier work of Kostant [7] and of Kostant-Kumar [8] on the Schubert calculus on $X$.

The splitting $\mathfrak{g}=\mathfrak{g}_{v}+\mathfrak{l}_{d}$ naturally defines a Lie bialgebra structure on $\mathfrak{g}_{v}$ and therefore a Poisson Lie group structure on $G_{v}$ [11. All the $G_{v}$-orbits in $\mathcal{L}$ become $G_{v}$-Poisson homogeneous spaces [3, 9]. We remark that in [1, Andruskiewitsch and Jancsa classified non-triangular Lie bialgebra structures on $\mathfrak{g}_{v}$ using BelavinDrinfeld triples. The one defined by the splitting $\mathfrak{g}=\mathfrak{g}_{v}+\mathfrak{l}_{d}$ comes from the standard Belavin-Drinfeld triple. We refer to [1] for details.

Example. Here we take $\mathfrak{g}=\mathfrak{s l}(2, \mathbb{C})$ and

$$
\mathfrak{g}_{v}=\mathfrak{s u}(1,1)=\left\{\left(\begin{array}{cc}
i x & y+i z \\
y-i z & -i x
\end{array}\right): x, y, z \in \mathbb{R}\right\} .
$$


Then $d=1$ and $\mathfrak{l}_{d}=\mathfrak{a}+\mathfrak{n}$ consists of upper triangular matrices in $\mathfrak{s l}(2, \mathbb{C})$ with real diagonal entries. Identify $G / B$ with $\mathbb{P}^{1}$ via the action

$$
\left(\begin{array}{ll}
a & b \\
c & d
\end{array}\right) \cdot\left[w_{0}: w_{1}\right]=\left[a w_{0}+b w_{1}: c w_{0}+d w_{1}\right]
$$

of $G$ on $\mathbb{P}^{1}$ and by taking $[1: 0] \in \mathbb{P}^{1}$ as the basepoint. There are two Bruhat cells: the zero-dimensional basepoint $[1: 0]$, and the other being the rest:

$$
U_{1}=\mathbb{P}^{1} \backslash\{[1: 0]\}=\left\{\left[w_{0}: w_{1}\right], w_{1} \neq 0\right\} .
$$

In terms of the holomorphic coordinate $z$ on $U_{1}$ given by $z=w_{0} / w_{1}$, the Poisson structure $\Pi_{v}$, up to a scalar multiple, is given by

$$
\Pi_{v}=i\left(1-|z|^{2}\right) \frac{\partial}{\partial z} \wedge \frac{\partial}{\partial \bar{z}} .
$$

Setting $u=1 / z$, we see that in the $u$-coordinate on the open set

$$
U_{0}=\left\{\left[w_{0}: w_{1}\right] \in \mathbb{P}^{1}, w_{0} \neq 0\right\}=\{[1: u], u \in \mathbb{C}\},
$$

we have

$$
\Pi_{v}=i\left(|u|^{2}-1\right)|u|^{2} \frac{\partial}{\partial u} \wedge \frac{\partial}{\partial \bar{u}}
$$

Thus $\Pi_{v}$ vanishes precisely at the basepoint $[1: 0]$ and at every point of the form $[z: 1]$ with $|z|=1$. If we identify $\mathbb{P}^{1}$ with the unit sphere $S^{2}$ in $\mathbb{R}^{3}$ via

$$
\mathbb{P}^{1} \longrightarrow S^{2}:\left[w_{0}, w_{1}\right] \longmapsto\left(\frac{2 \operatorname{Re}\left(w_{0} \overline{w_{1}}\right)}{\left|w_{0}\right|^{2}+\left|w_{1}\right|^{2}}, \frac{2 \operatorname{Im}\left(w_{0} \overline{w_{1}}\right)}{\left|w_{0}\right|^{2}+\left|w_{1}\right|^{2}}, \frac{\left|w_{0}\right|^{2}-\left|w_{1}\right|^{2}}{\left|w_{0}\right|^{2}+\left|w_{1}\right|^{2}}\right)
$$

then we see that $\Pi_{v}$ vanishes at the "North pole" $(0,0,1)$ and at every point on the Equator $x_{3}=0$. Under this identification, there are exactly three orbits of $\mathrm{SU}(1,1)$ on $S^{2}$ : the Northern hemisphere, the Equator, and the Southern hemisphere. Each of these three orbits is clearly a Poisson submanifold.

\section{Symplectic leaves of $\Pi_{v}$ IN $X$}

Suppose that $\mathcal{O}$ is a $G_{v}$-orbit in $X$ and $\mathcal{C}$ is a Bruhat cell such that $\mathcal{O} \cap \mathcal{C} \neq \emptyset$. Since $\mathfrak{g}=\mathfrak{g}_{v}+\mathfrak{l}_{d}, \mathcal{O}$ and $\mathcal{C}$ intersect transversally. By Proposition 3.4, $\mathcal{O} \cap \mathcal{C}$ is a Poisson submanifold of $\Pi_{v}$. In this section we show that $\left(\mathcal{O} \cap \mathcal{C}, \Pi_{v}\right)$ is a regular Poisson manifold, and we compute the dimension of its symplectic leaves.

It is well known [14] that there are only finitely many $G_{v}$-orbits in $X$. We first recall from [12, Section 6] some facts about these orbits.

Let $N_{G}(\mathfrak{h})$ be the normalizer subgroup of $\mathfrak{h}$ in $G$. Set

$$
\mathcal{Z}=\left\{g \in G: g^{-1} \tau_{v}(g) \in N_{G}(\mathfrak{h})\right\}
$$

Then $H$ acts on $\mathcal{Z}$ from the right by right multiplication, and $G_{v}$ acts on $\mathcal{Z}$ from the left by left multiplication. Let $Z$ be the double coset space

$$
Z=G_{v} \backslash \mathcal{Z} / H
$$

For each $z \in Z$, choose any $g_{z} \in \mathcal{Z}$ in the double coset $z$ and define $\mathcal{O}_{z}$ to be the $G_{v^{-}}$ orbit in $X$ through $g_{z} B \in X \cong G / B$. Clearly, $\mathcal{O}_{z}$ is independent of the choice of $g_{z}$. According to [12, Theorem 6.1.4], the map $z \mapsto \mathcal{O}_{z}$ is a one-to-one correspondence between the set $Z$ and the set of $G_{v}$-orbits in $X$. Let $W=N_{G}(\mathfrak{h}) / H$ be the Weyl group. Thus we also have the map

$$
\varphi: Z \longrightarrow W: z=G_{v} g_{z} H \longmapsto g_{z}^{-1} \tau_{v}\left(g_{z}\right) H \in W .
$$


According to [12, Theorem 6.4.2], the codimension of the $G_{v}$-orbit $\mathcal{O}_{z}$ in $X$ equals $l(\varphi(z))$, where $l$ is the length function on the Weyl group $W$. We also introduce the map

$$
\sigma_{z}=\varphi(z) \tau_{v}: \mathfrak{h} \longrightarrow \mathfrak{h} .
$$

For any $g_{z}$ in the double coset $z$, we also have $\sigma_{z}=\operatorname{Ad}_{g_{z}}^{-1} \circ \tau_{v} \circ \operatorname{Ad}_{g_{z}}$, so $\sigma_{z}$ is an involution.

Assume now that $z \in Z$ and $w \in W$ are such that $\mathcal{O}_{z} \cap \mathcal{C}_{w} \neq \emptyset$, where $\mathcal{C}_{w}$ is the Bruhat cell in $X$ corresponding to $w$, i.e. the $N$-orbit through $w \in G / B$. Then $\operatorname{dim}_{\mathbb{R}} \mathcal{C}_{w}=2 l(w)$, and since $\mathcal{O}_{z}$ and $\mathcal{C}_{w}$ intersect transversally, we have

$$
\operatorname{dim}\left(\mathcal{O}_{z} \cap \mathcal{C}_{w}\right)=2 l(w)-l(\varphi(z)) .
$$

Now define

$$
\delta_{z, w}=\operatorname{dim}\left(\mathfrak{h}^{w \sigma_{z} w^{-1}} \cap \mathfrak{h}^{-\tau_{v}}\right) .
$$

Theorem 4.1. Each symplectic leaf in the intersection $\mathcal{O}_{z} \cap \mathcal{C}_{w}$ has dimension equal to

$$
\operatorname{dim}\left(\mathcal{O}_{z} \cap \mathcal{C}_{w}\right)-\delta_{z, w}=2 l(w)-l(\varphi(z))-\delta_{z, w} .
$$

Proof. We use Proposition 3.2 to compute dimensions of the symplectic leaves in $\mathcal{O}_{z} \cap \mathcal{C}_{w}$. Let $x=g_{z} B \in X$ be a point in $\mathcal{O}_{z} \cap \mathcal{C}_{w}$, where $g_{z} \in \mathcal{Z}$ lies in the double coset $z$. Let $\mathfrak{l}_{x}=\operatorname{Ad}_{g_{z}}(\mathfrak{t}+\mathfrak{n}) \in \mathcal{L}$. Let $\mathfrak{n}\left(\mathfrak{l}_{x}\right)=\mathfrak{g}_{v} \cap \operatorname{Ad}_{g_{z}}(\mathfrak{h}+\mathfrak{n})$ be the normalizer subalgebra of $\mathfrak{l}_{x}$ in $\mathfrak{g}_{v}$, let $\mathfrak{m}\left(\mathfrak{l}_{x}\right)$ be the annihilator subspace of $\mathfrak{n}\left(\mathfrak{l}_{x}\right)$ in $\mathfrak{l}_{x}$, and let $\mathcal{V}\left(\mathfrak{l}_{x}\right)=\mathfrak{n}\left(\mathfrak{l}_{x}\right)+\mathfrak{m}\left(\mathfrak{l}_{x}\right)$. We claim that $\mathcal{V}\left(\mathfrak{l}_{x}\right)=\operatorname{Ad}_{g_{z}}\left(\mathfrak{h}^{\sigma_{z}}+\mathfrak{n}\right)$. Indeed, it follows from the definition of $\sigma_{z}$ that

$$
\operatorname{Ad}_{g_{z}}\left(\mathfrak{h}^{\sigma_{z}}\right) \subset \mathfrak{g}_{v} \cap \operatorname{Ad}_{g_{z}}(\mathfrak{h}+\mathfrak{n})=\mathfrak{n}\left(\mathfrak{l}_{x}\right) .
$$

It is also clear that $\operatorname{Ad}_{g_{z}} \mathfrak{n} \subset \mathfrak{m}\left(\mathfrak{l}_{x}\right)$, so

$$
\operatorname{Ad}_{g_{z}}\left(\mathfrak{h}^{\sigma_{z}}+\mathfrak{n}\right) \subset \mathfrak{n}\left(\mathfrak{l}_{x}\right)+\mathfrak{m}\left(\mathfrak{l}_{x}\right)=\mathcal{V}\left(\mathfrak{l}_{x}\right) .
$$

Since both $\operatorname{Ad}_{g_{z}}\left(\mathfrak{h}^{\sigma_{z}}+\mathfrak{n}\right)$ and $\mathcal{V}\left(\mathfrak{l}_{x}\right)$ have the same dimension, they must coincide.

Now let $S_{x}$ be the symplectic leaf of $\Pi_{v}$ in $X$ through $x$. By Proposition 3.2 . the codimension of $S_{x}$ in $\mathcal{O}_{z}$ is equal to $\operatorname{dim}\left(\mathcal{V}\left(\mathfrak{l}_{x}\right) \cap \mathfrak{l}_{d}\right)$. Let $\dot{w} \in N_{G}(\mathfrak{h})$ be a representative of $w$ in $K$. Since $x \in \mathcal{C}_{w}$, there exist $n \in N$ and $b \in B$ such that $g_{z}=n \dot{w} b$. Then we have

$$
\begin{aligned}
\mathcal{V}\left(\mathfrak{l}_{x}\right) \cap \mathfrak{l}_{d} & =\left(\operatorname{Ad}_{n \dot{w} b}\left(\mathfrak{h}^{\sigma_{z}}+\mathfrak{n}\right)\right) \cap\left(\mathfrak{h}^{-\tau_{v}}+\mathfrak{n}\right) \\
& =\operatorname{Ad}_{n}\left(\left(\operatorname{Ad}_{\dot{w}}\left(\mathfrak{h}^{\sigma_{z}}+\mathfrak{n}\right)\right) \cap\left(\mathfrak{h}^{-\tau_{v}}+\mathfrak{n}\right)\right) \\
& =\operatorname{Ad}_{n}\left(\mathfrak{h}^{w \sigma_{z} w^{-1}} \cap \mathfrak{h}^{-\tau_{v}}+\left(\operatorname{Ad}_{\dot{w}} \mathfrak{n}\right) \cap \mathfrak{n}\right),
\end{aligned}
$$

where in the last line we have the direct sum of vector spaces. Since

$$
\operatorname{dim}\left(\operatorname{Ad}_{\dot{w}} \mathfrak{n}\right) \cap \mathfrak{n}=\operatorname{dim}_{\mathbb{R}} X-\operatorname{dim}_{\mathbb{R}} \mathcal{C}_{w},
$$

we have

$$
\operatorname{dim}\left(\mathcal{V}\left(\mathfrak{l}_{x}\right) \cap \mathfrak{l}_{d}\right)=\delta_{z, w}+\operatorname{dim}_{\mathbb{R}} X-\operatorname{dim}_{\mathbb{R}} \mathcal{C}_{w}
$$

and thus

$$
\operatorname{dim} S_{x}=\operatorname{dim} \mathcal{O}_{z}-\operatorname{dim}\left(\mathcal{V}\left(\mathfrak{l}_{x}\right) \cap \mathfrak{l}_{d}\right)=\operatorname{dim}\left(\mathcal{O}_{z} \cap \mathcal{C}_{w}\right)-\delta_{z, w}
$$


Note that the number $\delta_{z, w}$ depends only on $d$ and the two Weyl group elements $\varphi(z)$ and $w$. Define $d: W \rightarrow W$ by $d(w)=\gamma_{d} w \gamma_{d}$. Following [12, we say that $w \in W$ is a $d$-twisted involution if $d(w)=w^{-1}$. Denote by $\mathcal{I}_{d}$ the set of all $d$-twisted involutions in $W$. Since for every $g \in G$ we have $\tau_{v}\left(g^{-1} \tau_{v}(g)\right)=\left(g^{-1} \tau_{v}(g)\right)^{-1}$, every $\varphi(z)$ is in $\mathcal{I}_{d}$. The Weyl group $W$ acts on $\mathcal{I}_{d}$ by

$$
w_{1} * w=w_{1} w d\left(w_{1}^{-1}\right) \text { for } w_{1} \in W \text {, and } w \in \mathcal{I}_{d},
$$

and the set $\varphi(Z) \subset \mathcal{I}_{d}$ is $W$-invariant. In fact, the $W$-action on $G / H$, given by $w \cdot g H=g w^{-1} H$, commutes with the left action of $G_{v}$ by left multiplication, and thus induces a left action of $W$ on $Z$, which we denote by $w \cdot z$ for $w \in W$ and $z \in Z$. It is also easy to see that $\varphi: Z \rightarrow W$ is $W$-equivariant, i.e. $\varphi(w \cdot z)=w * \varphi(z)$ for all $w \in W$ and $z \in Z$. Similarly, the involution $\tau_{v}: G \rightarrow G$ gives rise to an involution on $Z$ which depends only on $d$. Denote this involution by $z \rightarrow d(z)$. Then we also have $\varphi(d(z))=d \varphi(z)=\varphi(z)^{-1}$. As maps on $\mathfrak{h}$, we see that $w \sigma_{z} w^{-1}=(w * \varphi(z)) \tau_{v}$. Thus we also have

$$
\delta_{z, w}=\operatorname{dim}\left(\mathfrak{h}^{(w * \varphi(z)) \tau_{v}} \cap \mathfrak{h}^{-\tau_{v}}\right) .
$$

Corollary 4.2. 1) When $w * \varphi(z)=1$, symplectic leaves of $\Pi_{v}$ in $\mathcal{O}_{z} \cap \mathcal{C}_{w}$ are precisely its connected components.

2) Every open orbit $\mathcal{O}_{z}$ has an open symplectic leaf $\mathcal{O}_{z} \cap \mathcal{C}_{w_{0}}$, where $w_{0}$ is the longest element in $W$.

3) If $d=1$, symplectic leaves in an open orbit $\mathcal{O}_{z}$ are precisely the connected components of intersections of Bruhat cells with $\mathcal{O}_{z}$.

Proof. 1) When $w * \varphi(z)=1$, we have $\delta_{z, w}=0$, so every symplectic leaf in $\mathcal{O}_{z} \cap \mathcal{C}_{w}$ is open in $\mathcal{O}_{z} \cap \mathcal{C}_{w}$.

2) Since $\mathcal{C}_{w_{0}}$ is dense in $X$, it intersects with every open orbit $\mathcal{O}_{z}$. Since an orbit $\mathcal{O}_{z}$ is open if and only if $\varphi(z)=1$, statement 2) follows from 1) and the fact that $w_{0}$ commutes with $d$. The fact that $\mathcal{C}_{w_{0}} \cap \mathcal{O}_{z}$ is connected follows from the observation that $\mathcal{O}_{z}$ is a connected open complex submanifold of $X$ and thus $\mathcal{O}_{z} \cap\left(X \backslash \mathcal{C}_{w_{0}}\right)$ is a divisor in $\mathcal{O}_{z}$.

3) follows directly from 1).

Now consider the group $H^{\tau_{v}}=H \cap G_{v}$. Since the centralizer of $\mathfrak{h}^{\tau_{v}}$ in $G_{v}$ also centralizes $\mathfrak{h}$, we see that $H^{\tau_{v}}$ is the Cartan subgroup of $G_{v}$ corresponding to the Cartan subalgebra $\mathfrak{h}^{\tau_{v}}$. Then according to [6, Proposition 7.90] the group $H^{\tau_{v}}$ is connected.

The Poisson structure $\Pi_{v}$ on $X$ is $H^{\tau_{v}}$-invariant. Indeed, let $R \in \bigwedge^{2} \mathfrak{g}$ be the element given in (3.1) for $\mathfrak{l}_{1}=\mathfrak{g}_{v}$ and $\mathfrak{l}_{2}=\mathfrak{l}_{d}$. We can also represent $R$ as $R=\sum_{i} \xi_{i} \wedge y_{i}$, where $\left\{y_{i}\right\}$ is a basis of $\mathfrak{g}_{v}$, and $\left\{\xi_{i}\right\}$ is the dual basis of $\mathfrak{l}_{d}$ with respect to the pairing between $\mathfrak{g}_{v}$ and $\mathfrak{l}_{d}$ given by $\langle$,$\rangle , the imaginary part of the$ Killing form on $\mathfrak{g}$. If $h \in H^{\tau_{v}}$, then $\left\{\operatorname{Ad}_{h} y_{i}\right\}$ is a basis of $\mathfrak{g}_{v}$, and $\left\{\operatorname{Ad}_{h} \xi_{i}\right\}$ is its dual basis. Thus $\operatorname{Ad}_{h} R=R$.

Let $z \in Z$ and $w \in W$ be such that $\mathcal{O}_{z}$ and $\mathcal{C}_{w}$ have a non-empty intersection, and let $x \in \mathcal{O}_{z} \cap \mathcal{C}_{w}$. Clearly, $H^{\tau_{v}}$ leaves $\mathcal{O}_{z} \cap \mathcal{C}_{w}$ invariant. Since the Poisson structure $\Pi_{v}$ is $H^{\tau_{v}}$-invariant, if $S_{x}$ is the symplectic leaf of $\Pi_{v}$ through $x$, then $h S_{x}:=\left\{h x_{1}: x_{1} \in S_{x}\right\}$ is the symplectic leaf of $\Pi_{v}$ through $h x$. Define

$$
F_{x}:=\bigcup_{h \in H^{\tau_{v}}} h S_{x} .
$$

Proposition 4.3. For any $x \in X$, the set $F_{x}$ is a connected component of $\mathcal{O}_{z} \cap \mathcal{C}_{w}$. 
Proof. It is easy to see that if $F_{x_{1}} \cap F_{x_{2}} \neq \emptyset$, then $F_{x_{1}}=F_{x_{2}}$. The statement would follow once we prove that $F_{x}$ is an open subset of $\mathcal{O}_{z} \cap \mathcal{C}_{w}$ for each $x$.

Let $x=g_{z} B \in \mathcal{O}_{z} \cap \mathcal{C}_{w}$ with $g_{z} \in \mathcal{Z}$ in the double coset $z$. For $y \in \mathfrak{h}^{\tau_{v}}$, let $X_{y}$ be the vector field on $X$ generating the action of $\exp (t y) \in H^{\tau_{v}}$ on $X$. We claim that $X_{y}(x) \in T_{x} S_{x}$ if and only if $y \in p\left(\mathfrak{h}^{(w * \varphi(z)) \tau_{v}}\right)$, where $p: \mathfrak{h} \rightarrow \mathfrak{h}^{\tau_{v}}$ is the projection with respect to the decomposition $\mathfrak{h}=\mathfrak{h}^{\tau_{v}}+\mathfrak{h}^{-\tau_{v}}$. Assume the claim. Then since the kernel of the map $p: \mathfrak{h}^{(w * \varphi(z)) \tau_{v}} \rightarrow \mathfrak{h}^{\tau_{v}}$ has dimension $\operatorname{dim}\left(\mathfrak{h}^{(w * \varphi(z)) \tau_{v}} \cap \mathfrak{h}^{-\tau_{v}}\right)=\delta_{z, w}$, the image of the map

$$
J_{x}: \mathfrak{h}^{\tau_{v}} \longrightarrow T_{x} \mathcal{O}_{z} / T_{x} S_{x}: y \longmapsto X_{y}(x)+T_{x} S_{x}
$$

has dimension equal to $\operatorname{dim}\left(\mathfrak{h}^{\tau_{v}}\right)-\operatorname{dim}\left(\mathfrak{h}^{(w * \varphi(z)) \tau_{v}}\right)+\delta_{z, w}=\delta_{z, w}$. Thus $J_{x}$ is onto, and the $H^{\tau_{v}}$-orbit in $\mathcal{O}_{z} \cap \mathcal{C}_{w}$ through $x$ is transversal to the symplectic leaf $S_{x}$. It follows that $F_{x}$ is open in $\mathcal{O}_{z} \cap \mathcal{C}_{w}$.

It remains to prove the claim. Also denote by $p: \mathfrak{g} \rightarrow \mathfrak{g}_{v}$ the projection with respect to the decomposition $\mathfrak{g}=\mathfrak{g}_{v}+\mathfrak{l}_{d}$, and let $q$ be the projection $q: \mathfrak{g}_{v} \rightarrow$ $\mathfrak{g}_{v} / \mathfrak{g}_{v} \cap \operatorname{Ad}_{g_{z}} \mathfrak{b} \cong T_{x} \mathcal{O}_{z}$. Then by [9, Corollary 7.3], we have $T_{x} S_{x}=(q \circ p)\left(\mathcal{V}\left(\mathfrak{l}_{x}\right)\right)$, where, as in the proof of Theorem 4.1, $\mathcal{V}\left(\mathfrak{l}_{x}\right)=\operatorname{Ad}_{g_{z}}\left(\mathfrak{h}^{\sigma_{z}}+\mathfrak{n}\right)$. Let $y \in \mathfrak{h}^{\tau_{v}}$. If $X_{y}(x) \in T_{x} S_{x}$, then there exist $y_{1} \in \mathfrak{l}_{d}$ and $y_{2} \in \mathfrak{g}_{v}$ with $y_{1}+y_{2} \in \mathcal{V}\left(\mathfrak{l}_{x}\right)$ such that $y-y_{2} \in \mathfrak{g}_{v} \cap \operatorname{Ad}_{g_{z}} \mathfrak{b} \subset \mathcal{V}\left(\mathfrak{l}_{x}\right)$. Thus $y+y_{1}=y-y_{2}+y_{1}+y_{2} \in \mathcal{V}\left(\mathfrak{l}_{x}\right)$. Write $y_{1}=\xi_{1}+u_{1}$, where $\xi_{1} \in \mathfrak{h}^{-\tau_{v}}$ and $u_{1} \in \mathfrak{n}$. Then there exist $\xi_{2} \in \mathfrak{h}^{\sigma_{z}}$ and $u_{2} \in \mathfrak{n}$ such that $y+\xi_{1}+u_{1}=\operatorname{Ad}_{g_{z}}\left(\xi_{2}+u_{2}\right)$. Write $g_{z}=n \dot{w} b$, where $n \in N, b \in B$, and $\dot{w}$ is a representative of $w$ in $K$. Write $\operatorname{Ad}_{n^{-1}}\left(y+\xi_{1}+u_{1}\right)=y+\xi_{1}+u_{1}^{\prime}$ and $\operatorname{Ad}_{b}\left(\xi_{2}+u_{2}\right)=\xi_{2}+u_{2}^{\prime}$, where $u_{1}^{\prime}, u_{2}^{\prime} \in \mathfrak{n}$. Then we have

$$
y+\xi_{1}+u_{1}^{\prime}=\operatorname{Ad}_{\dot{w}}\left(\xi_{2}+u_{2}^{\prime}\right) \text {. }
$$

Since $y+\xi_{1}, \operatorname{Ad}_{\dot{w}} \xi_{2} \in \mathfrak{h}$ and $u_{1}^{\prime}, \operatorname{Ad}_{\dot{w}} u_{2}^{\prime} \in \mathfrak{n}+\mathfrak{n}_{-}$, where $\mathfrak{n}_{-}=\theta(\mathfrak{n})$, we have $y+\xi_{1}=\operatorname{Ad}_{\dot{w}} \xi_{2} \in \mathfrak{h}^{(w * \varphi(z)) \tau_{v}}$. Thus $y \in p\left(\mathfrak{h}^{(w * \varphi(z)) \tau_{v}}\right)$. Conversely, if $y \in \mathfrak{h}^{\tau_{v}}$ is such that $y+\xi_{1} \in \mathfrak{h}^{(w * \varphi(z)) \tau_{v}}=\operatorname{Ad}_{\dot{w}} \mathfrak{h}^{\sigma_{z}}$ for some $\xi_{1} \in \mathfrak{h}^{-\tau_{v}}$, write $y+\xi_{1}=\operatorname{Ad}_{\dot{w}} \xi_{2}$ for $\xi_{2} \in \mathfrak{h}^{\sigma_{z}}$. Let $\operatorname{Ad}_{b^{-1}} \xi_{2}=\xi_{2}+u_{2}$ for some $u_{2} \in \mathfrak{n}$. We then have

$$
\operatorname{Ad}_{n}\left(y+\xi_{1}\right)=\operatorname{Ad}_{n \dot{w} b}\left(\xi_{2}+u_{2}\right) \in \mathcal{V}\left(\mathfrak{l}_{x}\right) \text {. }
$$

On the other hand, let $\operatorname{Ad}_{n}\left(y+\xi_{1}\right)=y+\xi_{1}+u_{1}$ with $u_{1} \in \mathfrak{n}$. We see that $y=p\left(\operatorname{Ad}_{n}\left(y+\xi_{1}\right)\right)$ so $X_{y}(x) \in T_{x} S_{x}$.

\section{INVARIANT POISSON COHOMOLOGY OF OPEN ORBITS}

Let $\mathcal{O}_{z}$ be a $G_{v}$-orbit in $X$ equipped with the Poisson structure $\Pi_{v}$. Then $\left(\mathcal{O}_{z}, \Pi_{v}\right)$ is a Poisson homogeneous space for the Poisson Lie group $G_{v}$. The $G_{v^{-}}$ invariant Poisson cohomology of $\left(\mathcal{O}_{z}, \Pi_{v}\right)$, denoted by $H_{\Pi_{v}, G_{v}}^{\bullet}\left(\mathcal{O}_{z}\right)$, is defined as the cohomology of the cochain complex $\left(\chi^{\bullet}\left(\mathcal{O}_{z}\right)^{G_{v}}, \partial_{\Pi_{v}}\right)$, where $\chi^{\bullet}\left(\mathcal{O}_{z}\right)^{G_{v}}$ is the space of all $G_{v}$-invariant complex multi-vector fields on $\mathcal{O}_{z}, d_{\Pi_{v}}(V)=\left[\Pi_{v}, V\right]$, and $[\cdot, \cdot]$ is the Schouten bracket of the multi-vector fields.

Proposition 5.1. When $\mathcal{O}_{z}$ is an open $G_{v}$-orbit in $X$, the $G_{v}$-invariant Poisson cohomology $H_{\Pi_{v}, G_{v}}^{\bullet}\left(\mathcal{O}_{z}\right)$ is isomorphic to the de Rham cohomology of $X$.

Proof. As in the proof of Theorem 4.1, let $x=g_{z} B \in X$ be an arbitrary point in $\mathcal{O}_{z}$, where $g_{z} \in \mathcal{Z}$ is in the coset $z$, and let $\mathcal{V}\left(\mathfrak{l}_{x}\right)=\operatorname{Ad}_{g_{z}}\left(\mathfrak{h}^{\sigma_{z}}+\mathfrak{n}\right)$. Since $\mathcal{O}_{z}$ is open, the stabilizer subalgebra of $\mathfrak{g}_{v}$ at $x$ is $\mathfrak{g}_{v} \cap \mathcal{V}\left(\mathfrak{l}_{x}\right)=\operatorname{Ad}_{g_{z}}\left(\mathfrak{h}^{\sigma_{z}}\right)$. By [9, Theorem 7.5], the $G_{v}$-invariant Poisson cohomology $H_{\Pi_{v}, G_{v}}^{\bullet}\left(\mathcal{O}_{z}\right)$ is isomorphic to the relative Lie algebra cohomology of the Lie algebra $\mathcal{V}\left(\mathfrak{l}_{x}\right) \otimes \mathbb{C}$ relative to the subalgebra 
$\left(\operatorname{Ad}_{g_{z}}\left(\mathfrak{h}^{\sigma_{z}}\right)\right) \otimes \mathbb{C}$. Thus the $G_{v}$-invariant Poisson cohomology is isomorphic to the $\mathfrak{h}$-invariant part of the Lie algebra cohomology of the direct sum Lie algebra $\mathfrak{n} \oplus \mathfrak{n}$ with coefficients in $\mathbb{C}$, which by Kostant's theorem [7, is isomorphic to the de Rham cohomology of $X$.

\section{REMARKS}

We have constructed a Poisson structure $\Pi_{v}$ on $X$ for each Vogan diagram $v$ for $\mathfrak{g}$ (which is not necessarily normalized). In particular, each Bruhat cell $\mathcal{C}_{w}$ in $X$ carries the Poisson structure $\Pi_{v}$. It would be interesting to study connections between the Poisson structures for different $v$. Especially interesting are the properties of $\Pi_{v}$ that depend only on the inner class $d$ of the real form $\mathfrak{g}_{v}$. We also remark that the Poisson structure $\Pi_{v}$ is defined on the whole variety $\mathcal{L}$ of Lagrangian subalgebras of $\mathfrak{g}$. We have only been looking at the restriction of $\Pi_{v}$ to a particular $G$-orbit, namely the $G$-orbit through the Lagrangian subalgebra $\mathfrak{t}+\mathfrak{n}$. There are many other interesting $G$-orbits in $\mathcal{L}$, such as the $G$-orbit through a given real form of $\mathfrak{g}$. It would be interesting to study the properties of the Poisson structure $\Pi_{v}$ on these orbits as well as on their closures, with respect to both the classical topology and the Zariski topology.

\section{ACKNOWLEDGMENTS}

Initial ideas for the paper came from discussions with Sam Evens. The first author was supported by NSF grant DMS-0072520. The second author was supported by NSF grants DMS-0105195 and DMS-0072551 and by the HHY Physical Sciences Fund at the University of Hong Kong. Both authors are grateful to IHES for hospitality and the second author also thanks Rencontres Mathématiques de Glanon 2003, where the paper was completed.

\section{REFERENCES}

[1] N. Andruskiewitsch and P. Jancsa. On simple real Lie bialgebras. Int. Math. Res. Not., 2004 (3), 139-158. MR2038773

[2] J. Adams and D. Vogan. L-groups, projective representations, and the Langlands classification. Amer. J. Math., 114(1):45-138, 1991. MR.1147719 (93c:22021)

[3] S. Evens and J.-H. Lu. On the variety of Lagrangian subalgebras, I. Ann. Scient. Éc. Norm. Sup., 34: 631-668, 2001. MR1862022(2002i:17014)

[4] S. Evens and J.-H. Lu. Poisson harmonic forms, Kostant harmonic forms, and the $S^{1}$ equivariant cohomology of K/T. Adv. Math., 142:171-220, 1999. MR1680047 (2001e:53085)

[5] S. Helgason. Differential geometry, Lie groups, and symmetric spaces. Pure and Applied Math., 80, Academic Press, 1978. MR0514561 (80k:53081)

[6] A. W. Knapp. Lie Groups beyond an Introduction. Second Edition. Progress in Math., 140, Birkhäuser, Boston, 2002. MR.1920389 (2003c:22001)

[7] B. Kostant, Lie algebra cohomology and generalized Schubert cells, Ann. of Math., 77 (1) (1963), 72-144. MR0142697(26:266)

[8] B. Kostant and S. Kumar. The nil Hecke ring and cohomology of $G / P$ for a Kac-Moody group G. Adv. Math., 62(3):187-237, 1986. MR0866159 (88b:17025b)

[9] J.-H. Lu. Poisson homogeneous spaces and Lie algebroids associated to Poisson actions. Duke Math. J., 86(2):261-304, 1997. MR1430434 (98d:58204)

[10] J.-H. Lu. Coordinates on Schubert cells, Kostant's harmonic forms, and the Bruhat Poisson structure on G/B. Transform. Groups, 4:355-374, 1999. MR 1726697(2001k:22018)

[11] J.-H. Lu and A. Weinstein. Poisson Lie groups, dressing transformations, and Bruhat decompositions. J. Diff. Geom., 31:501-526, 1990. MR1037412 (91c:22012)

[12] R. W. Richardson and T. A. Springer. Combinatorics and geometry of $K$-orbits on flag manifolds. Contemporary Mathematics, Vol. 153, 109-142, 1993. MR.1247501 (94m:14065) 
[13] Ya. Soibelman. Algebra of functions on a compact quantum group and its representations. Leningrad Math. J. 2:161-178, 1991. MR1049910(91i:58053a)

[14] J. A. Wolf. The action of a real semisimple Lie group on a complex flag manifold, I: Orbit structure and holomorphic arc components. Bull. Amer. Math. Soc., 75:1121-1237, 1969. MR0251246 (40:4477)

Department of Mathematics, University of Arizona, Tucson, Arizona 85721-0089

E-mail address: foth@math.arizona.edu

Department of Mathematics, The University of Hong Kong, Pokfulam, Hong Kong

E-mail address: jhlu@maths.hku.hk 